\title{
Blood-based markers for T2DM
}

Epigenetic alteration of genes occurs during ageing, and these changes can be detected in leukocytes. Interestingly, some of these alterations are also associated with insulin secretion; these findings, reported in Nature Communications, might be used as the basis for a blood-based predictive test for islet-cell dysfunction.

"We found that ageing is associated with increased DNA methylation of specific genes in both human pancreatic islets and blood," explains Charlotte Ling, who led the study. The team found methylation sites that were associated with increasing age in 154 genes and intergenic regions in islet samples from donors who did not have diabetes mellitus (aged 26-74 years). Of these genes, 30 had previously been associated with diabetes mellitus or dysfunctional islets, $\beta$ cells or mitochondria. The researchers then identified the transcripts with expression changes that were associated with methylation levels and could be detected in both leukocytes and pancreatic islets.

Using blood samples from the Danish Family Study, Ling and her team found that increased methylation of certain genes (KLF14 and ZNF518B) was significantly associated with elevated insulin levels. These data suggest that the epigenetic markers that can be detected in blood might be used to predict future capacity for insulin secretion.

Finally, the investigators used blood samples from another study in which the participants were healthy at enrolment and were monitored for the development of type 2 diabetes mellitus (T2DM) over a mean period of 10.8 years. Importantly, increased methylation levels of some genes (KLF14, FHL2 and GNPNAT1) was associated with a reduced risk of developing T2DM.

"The associations of our epigenetic biomarkers with future T2DM were of similar magnitude as for some of the top SNPs from genome-wide association studies," clarifies Ling. But she cautions that, "the predictive potential of these single biomarkers is not strong enough to warrant clinical use and more work is needed to identify additional markers."

Tim Geach

ORIGINAL ARTICLE Bacos, K. et al. Blood-based biomarkers of age-associated epigenetic changes in human islets associate with insulin secretion and diabetes. Nat. Commun. 7, 11089 (2016) 